Meta

Journal des traducteurs

Translators' Journal

\title{
Index des mots et sujets traités dans le volume $\mathbf{2 0}$
}

Volume 20, numéro 4, décembre 1975

URI : https://id.erudit.org/iderudit/003202ar

DOI : https://doi.org/10.7202/003202ar

Aller au sommaire du numéro

Éditeur(s)

Les Presses de l'Université de Montréal

ISSN

0026-0452 (imprimé)

1492-1421 (numérique)

Découvrir la revue

Citer ce document

(1975). Index des mots et sujets traités dans le volume 20. Meta, 20(4), 336-342.

https://doi.org/10.7202/003202ar d'utilisation que vous pouvez consulter en ligne.

https://apropos.erudit.org/fr/usagers/politique-dutilisation/ 


\section{INDEX DES MOTS ET SUJETS TRAITES DANS LE VOLUME 20}

Abrègement, 4, p. 275, 276.

Abréviation, 4, p. $271,275$.

Absence de prestige de la traduction,

$$
1, \text { p. } 50 .
$$

Absence de recherche en traduction, 1, p. 54

Acadie, 4, p. 328.

Accélération du débit, 4, p. 58 .

Accent circonflexe, 4, p. 281.

Accord anaphorique, 4, p. 269.

Acier, 2, p. 167.

A coups, 4, p. 310

-acre, 4, p. 264.

Acronyme, 4, p. 275.

Acronymes alphabétiques, 4 , p. 275

Acronymes phonétiques, 4 , p. 275.

Acronymes syllabiques, 4, p. 275.

Acte de traduction, 1, p. 11 .

Addition, 4, p. 319

Affaires étrangères, 1, p. 81.

Affichage, 1, p. 82.

Agente, 4, p. 267

Aide, 2, p. 155

Aide financière, 2, p. 154.

A.l.I.C., 1, p. $37 ; 2$, p. 174

Aire de réception, 4, p. 311.

Alcootest, 1, p. 31 ; 2, p. 158

Allure, 4, p. 310.

Allocation, 2, p. 155.

Amateur, 1, p. 45

Analyse textuelle, 2, p. 166.

Anglicisation, 1, p. 28.

Anglicismes, 1, p. 82.

Anglicismes au Québec, 1, p. 29.

Anglicismes en France, 1, p. 29.

Antibruit, 2, p. 170 .

Appel de fonds, 3, p. 227.

"Application », 1, p. 30.

Apports, 4, p. 318.

Apprendre à traduire, 1, p. 46.

Appuis, 4, p. 311.

Aptitude, 3, p. 219.

Aptitudes du traducteur, 2, p. 137

Arbitrage, 3, p. 227.

Archéologie, 3, p. 237.

Architecture, 2, p. 170.

Argus, 1, p. 75 .

Arrière-plan socio-culturel, 2, p. 164.

Arrivée, 4, p. 309.

Arrivée dans un mouchoir, 4, p. 309.

Arts ménagers, 1, p. 81 .

Asphalte, 3, p. 209.

Assemblée, 1, p. 28.

Assesseur, 1, p. 31.

Assistance, 2, p. 155.

Association canadienne des traducteurs diplômés, 1, p. 75 .

Association des diplômés de l'Institut de traduction de l'Université de Montréal,

1, p. 75 .
Association internationale des interprètes de conférence, 1, p. 79.

Associations professionnelles, 1, p. 68

Association technologique de langue

française d'Ottawa, 1, p. 75.

Assurances sociales, 1, p. 30.

Asymétrie de la traduction, 2, p. 165.

Athlétisme, 4, p. 308.

-ation, 4, p. 264

Attitudes intellectuelles, 2, p. 137.

Attitudes psychologiques, 2, p. 137.

Auditeur, 2, p. 156.

Auto-école, 1, p. 31.

Automatique interurbain, 1, p. 32.

Automatisation du tri, 2, p. 150.

Automobile, 1, p. 31

"Aviseur légal », 1, p. 30.

Avoirs financiers, 4, p. 317

A vos marques, 4 , p. 309 .

Bac, 4, p. 312 .

Banque de terminologie, 1 , p. $59,66,71$; 2 , p. $131,133,139 ; 4$, p. $330,332$.

Barre, 4, p. 311

Bâtiment, 1, p. $31 ; 2$, p. 170.

"Bâtisse ", 1, p. 31 .

Bâtonnet fluorescent, 2, p. 151.

«Bénéfices marginaux $~, 1$, p. 30.

"Bénéfices sociaux ", 1, p. 30.

Bibliographie, 3, p. 239.

Bibliographie de l'architecture, du bâtiment et des travaux publics, 2 , p. 170

Bibliographie de la traduction, 1, p. 23

Bibliographie (dérive des continents )

4, p. 316

Bibliographie des chroniques de langage, 4, p. 321.

Bibliographie des livres canadiens en traduction, 1 , p. 83 .

Bibliographie des sigles, 4, p. 302.

"Bien-être », 1, p. 30 .

Biennale de Chicoutimi, 2, p. $174 ; 4$, p. 327.

Biennale de langue française, 2, p. 175 ; 4 , p. 330 .

Bienvenue, 1, p. 30.

Bilinguisme, 1, p. 12,$109 ; 4$, p. 322.

Bit fluorescent, 2, p. 151.

Bitume, 3, p. 208 .

Bivalence des attitudes, 3, p. 195.

Bon finisseur, 4 , p. 310 .

Bourse et commerce des valeurs mobilières, 3, p. 225.

Bouteille consignée, 1, p. 30.

Brachigraphie, 4, p. 271.

Bruit parasite, 2, p. 170.

Bureau, 1, p. 30.

Butoir, 4, p. 312.

Cales, 4, p. 309.

Canadien français, 1 , p. 33

Canadien français et traduction, 1, p. 63. 
"Cap de roue 》, 1, p. 31.

Capitalisation, 4, p. 287.

Capitalisation des sigles, 4 , p. 283.

Caractère technique des traductions, 1, p. 58.

Caractéropathies, 1, p. 81.

Cartouche, 4, p. 266

Cartulaire, 4, p. 282

Cassé, 4 , p. 309 .

Catégorie grammaticale, 4, p. 264.

Cegep, 1, p. 30.

Cégépien, 1, p. 30.

Cendrée (en), 4, p. 308

Cent dix mètres haies, 4, p. 310.

Centre commercial, 1, p. 30.

"Centre d'achats", 1, p. 30.

Centre d'information documentaire, 2, p. 133.

Cercle, 4, p. 312.

Cercle des traducteurs; 1, p. 76.

"Chambre ", 1, p. 30.

"Char, 1, p. 31

Charabia infâme, 4 , p. 322

Charnières bilingues, 1, p. 30 .

"Charrue ", 1, p. 29.

Chaussures à pointes, 4, p. 312.

Cheval, 1, p. 81

Chrono, 4, p. 309.

Chronométrage électrique, 4, p. 309.

Chronomètres, 4, p. 309.

Chronométreur, 4, p. 309.

C.I.D., 1, p. 31 .

Ciseau des jambes, 4 , p. 311

Cité, 1, p. 33

Civilisation et traduction, 1, p. 12

Claquage, 4, p. 308

Claquer (se) un muscle, 4 , p. 308

Classement alphabétique des sigles, 4, p. 277.

Classification selon l'optique, 3, p. 198.

Clichés tirés de la Bible, 4, p. 324.

Clichés tirés de Shakespeare, 4, p. 324.

Comité intersociétés, 1, p. 78 .

Comité technique, $\mathrm{n}^{\circ} 37,4$, p. 332.

Commissaire aux comptes, 2 , p. 156

"Commission des liqueurs" $1, \mathrm{p}, 33$.

Commissaire de sociétés, 2, p. 156.

Commune, 1, p. 31.

Communication, 2, p. 164.

Communication et interprétation, 1, p. 37

Complet, 1, p. 30

Composer, 1, p. 31 .

Composition interurbaine directe, 1, p. 31

Compte, 4 , p. 319.

Concentration des interprètes, 1, p. 38

Concours, 4, p. 308, 311 .

Concrétivité, 3, p. 195.

Concurrent, $4,0.308$.

Congrès des traducteurs, 1, p. 75 .

Connaissances du traducteur, 2, p. 137.

Connaissance formelle de la langue, 1, p. 49.

Conscience professionnelle, 1, p. 50.

Conseil d'administration, 1 , p. 30

Conseiller juridique, 1 , p. 30.

Contentieux, 1, p. 30 .

Contraction, 4 , p. 281.

Contrat type, 3 , p. 246

Contre-anglicisme, 1, p. 29.

Contrôle comptable, 2, p. 157.

Contrôle de gestion, 2, p. 157

Contrôle interne, 2, p. 156.

Contrôle tournant, 2, p. 156.
Contrôleur, 2, p. 156

Conventions collectives, 1, p. 81 .

Coopération en terminologie, 1, p. 67.

Coordonnées, 2 , p. 135

Copule être, 4, p. 265.

Corde (à la ), 4, p. 309

Corporation, 1, p. 33.

Corporation des traducteurs et interprètes du Nouveau-Brunswick, 1, p. 78.

Corporation des traducteurs professionnels du Québec, 1, p. 76.

"Correct », 1, p. 29.

Couloirs, 4, p. 308.

Coup de feu, 4 , p. 309.

Coup de rein, 4 , p. 310

Couple, 4, p. 266.

Coups de pointe, 4 , p. 310.

Courbe, 4, p. 309.

Coureurs de demi-fond, 4 , p. 310 .

Courses, 4 , p. 308

Courses de demi-fond, 4, p. 309.

Courses de fond, 4, p. 309.

Courses de relais, 4, p. 309.

Courses de vitesse, 4 , p. 309

Courses d'obstacles, 4, p. 309, 310.

Courses plates, 4 , p. 310 .

Couture, 1, p. 81 .

Couvaison, 2, p. 170.

Création publicitaire, 1, p. 65

Créativité, 3, p. 219.

Crispation, 4, p. 310.

Crisper (se), 4, p. 310

Critères d'évaluation des étudiants, 1, p. 54.

Critères d'évaluation des programmes en traduction, 1, p. 54.

Critique de la traduction, 3, p. 236.

Culotte, 4 , p. 312.

Culture générale, 1, p. 60

C.T.I.C., 2, p. 174.

Cycle, 1 , p. 31 .

«Débenture », 3, p. 227.

Décathlon, 4 , p. 312

Déclarer forfait, 4, p. 309.

Déclasser, 4, p. 311.

Définition de la traduction, 1, p. 62 .

Déformation de sens des emprunts, 1, p. 29.

Défrancisation de la population, 1, p. 59.

Délai d'adaptation d'un étudiant au travail, 1, p. 45 .

Démagogie de la facilité, 1, p. 61 .

Demi (notation des heures), 1, p. 32.

Demi-finale, 4 , p. 309.

Départ accroupi, 4, p. 309.

Départager aux essais, 4, p. 312.

Départ allongé, 4, p. 309

Départ debout, 4, p. 310.

"Département légal ", 1 , p. 30

Départ en bascule, 4, p. 309

Départ en décalage, 4 , p. 309.

Départ groupé, 4 , p. 309.

Dérivation, 1, p. 80.

Dérive des continents, 4, p. 313.

Dernier parcours, 4 , p. 311 .

Deuxième congrès des traducteurs, 1, p. 76.

Dialecte hippie, 4 , p. 324 .

Dialecte noir, 4, p. 324

Dictionnaires bilingues, 2 , p. 130

Dictionnaires d'abréviation's, 4, p. 273. 
Dictionnaires des difficultés de la traduction, 2, p. 133

Didactique de la traduction, 2, p. 166.

Dimension sociale de la vie professionnelle, I, p. 68.

Discipline, 4, p. 308.

Discours, 1, p. 14.

Discours glossolaliste, 1, p. 109.

Discours psychotique, 1, p. 109.

Discours schizophrénique, 1, p. 109.

Doctoresse, 4, p. 267.

Dominance, 3, p. 194

Dons et aptitudes du traducteur, 2, p. 317.

Dossard, 4, p. 312.

Dotations en capital, 4, p. 318

Double, 4, p. 309.

Doublé, 4, p. 309.

Double ciseau, 4 , p. 311.

Drogues, 1 , p. 81 .

Droit civil, 1, p. 48.

Droits d'auteur, 1, p. 30.

Echanges internationaux, 4, p. 330.

Echauffer (s'), 4, p. 308 .

"Ecole de conduite", 1, p. 31.

"Ecole des gradués », 1, p. 31.

Ecole privée de traduction, 1, p. 61

Economie linguistique, 4, p. 265.

Ecriture, 1, p. 14.

Edifice, 1, p. 31

Effectif des interprètes, 1, p. 36.

Effectifs étudiants en traduction, 1, p. 52.

"Eligible " 1, p. 35 .

Ellipse, 4, p. 276

Emploi des points dans les sigles, 4, p. 285.

Emploi des sigles, 4, p. 293.

Emplois et fonctions, 4, p. 269.

«Employé postal », 2, p. 150.

Emprunt, 2, p. 133.

Emprunt de conversion, 3, p. 228.

Emprunt obligatoire, 3, p. 226.

Encouragement, 2, p. 155

Enfance inadaptée, 1, p. 81.

"Engin 》, 1, p. 29.

Engin, 4, p. 312.

"Enregistrement des véhicules », 1, p. 33.

Enseignement alterné, 1, p. 56.

Enseignement de la traduction, 1, p. 17, 42 .

Enseignement des langues de spécialités, 2 , p. 166.

Enseignement par les professionnels,

$1, \mathrm{p}, 64$.

Entreprise et traduction, 3, p. 240.

Environnement, 2, p. 169.

Epargner, 1, p. 28

Epicène, 4, p. 266.

Epreuves, 4, p. 308

Epreuves (tarif), 4, p. 327.

Equipes mixtes de traduction, 1, p. 60.

Escrime, 2, p. 141.

Essence, 1, p. 31.

Etre enfermé, 4, p. 310.

Etalonner, 4, p. 311.

Espionnage (abréviations), 4, p. 296.

Et dans les nombres, 1, p. 28.

Etat de compte, 4, p. 319.

Etat, 4, p. 319.

Etudes lexicales, 4 , p. 322

Evaluation extra-comptable, 2, p. 156

Evolution de l'écriture des sigles, 4, p. 283.
Evolution de la traduction, 1, p. 58.

Evolution des théories de la traduction,

1, p. 7.

Evolution du français au Canada, 2, p. 140.

Evolution linguistique, 4 , p. 321 .

Ex aequo, 4, p. 312 .

Exclusivité de l'acte, 1, p. 77

Exclusivité du titre, 1, p. 77.

Exercices d'assouplissement, 4, p. 308.

Exhaustion, 4, p. 321.

Expert-comptable, 2, p. 157.

Exprès (par), 1, p. 32 .

«Extension », 1, p. 32.

"Facteur causal ", 2, p. 134

Facteurs de l'inflation lexicale, 2, p. 131

Factrice, 4, p. 270

Facture, 4, p. 318.

Faire cavalier seul, 4 , p. 310

Faux départ, 4, p. 309.

Fédération internationale des traducteurs, 1, p. 76.

Faire le plein, 1, p. 31.

Faits de culture, 1, p. 12.

Faits de discours, 1, p. 14.

Fendre (se), 2, p. 142.

Fente, 2, p. 142 .

Fiches terminologiques, 3, p. 181.

Fichiers automatiques, 1 , p. 60 .

Fidélité, 2, p. 139.

Fidélité de gaze, 4, p. 257.

Fil, 4, p. 312.

Fil de chaîne, 3, p. 237

Fil de trame, 3, p. 237.

Filière d'avancement, 3, p. 220.

Filière d'engagement, 3 , p. 220 .

Filière de promotion, 3 , p. 220

Fonctions du terminologue d'entreprise, 1, p. 73.

Fonds propres, 4, p. 317.

Force centrifuge, 4, p. 312

Formation continue, 1, p. 53.

Formation des sigles, 4, p. 283.

Formation de stocks, 4, p. 317.

Formation des traductions, 1, p. 60 ; 2 , p. $165 ; 3$, p. 180 .

Formation des traducteurs anglophones, 1, p. 61.

Formation des traducteurs littéraires, 2, p. 166.

Formation du jugement, 1, p. 47.

Formation du traducteur et de l'interprète, 1, p. 112.

Formation générale, 1, p. 47.

Formation spécialisée, 1, p. 47.

Formes invariables, 4, p. 266.

Formes variables, 4, p. 267.

Fosse de réception, 4, p. 311.

Foulée, 4, p. 310.

Foulée (dans la), 4, p. 310.

Fractionnement de poste, 3, p. 220.

Frais virés (à), 1, p. 32.

Français au Canada, 1, p. $28 ; 4$, p. 321, 328.

Français québécois, 1, p. 28.

Franchir, 4, p. 312.

Franchir la haie, 4, p. 311

Francisation des entreprises, 4, p. 329.

Franco-Ontariens, 4, p. 328

Franco-Québécois, 1, p. 33. 
"Frapper", 1, p. 29.

"Gaz", 1, p. 31 .

Généraliste / spécialiste, 1, p. 60

Genre (étude du), 4, p. 263.

Genre grammatical, 4, p. 263, 268.

Genre lié au sexe, 4, p. 268.

Genre naturel, 4, p. 263, 268.

Genre précisé, 4, p. 267.

Gens (accord), 4, p. 266.

Gens de maison, 4, p. 266

Gérant/directeur, 1, p. 30

Gestion, 3, p. 218

Glace, 1, p. 80 .

Glissement du nom vers l'adjectif, 2, p. 133.

Glossaire, 2, p. 167

Glossaire de décodage, 2, p. 131.

Gouvernement fédéral et francophonie canadienne, 4 , p. 328.

"Gouverneurs ", 1, p. 30.

«Gradué », 1, p. 31 .

Grammaire systématique, 3, p. 241.

Greffe, 4, p. 266.

Groupe létrique à valeur alphabétique, 4 , p. 284

Groupe létrique à valeur syllabique, 4, p. 284.

Guide de travail en terminologie, 3, p. 225.

"Habit ", 1, p. 29.

Hausser, 1, p. 28

"Heure d'affaires », 1, p. 30.

Heures d'ouverture, 1, p. 30

Heurter un obstacle, 4, p. 310.

Hexagonal, 2, p. 135 .

Hippie, 4, p. 324.

Historique de l'enseignement de la traduction, 1, p. 43 .

Historique de Meta, 1, p. 5.

Historique des sociétés professionnelles, 1, p. 75 .

Homonymie, 4, p. 266.

Hres, 1, p. 32.

Humain, 2, p. 134.

Hypostase, 2, p. 134

Hypostasier, 2, p. 135

Identification des sigles, 4, p. 293

Idéologie du " laxisme ", 1, p. 62.

Immatriculation des véhicules, 1, p. 33.

Impossibilité de la spécialisation, 2, p. 130

Impót sur le revenu, 1, p. 81 .

Improductivité, 3, p. 219.

Inanimé, 2, p. 134 .

Incapacité, 3, p. 219.

Incapacité de penser de façon analytique, 1 , p. 49.

Incontinence lexicale, 2, p. 131.

Indéfinis, 4, p. 265.

Indemnité, 2 , p. 155 .

Indépendance du Québec et la traduction, 1, p. 59,61 .

Indexation, 2, p. 151.

Index photoluminescent, 2, p. 151.

Indicatif postal, 2, p. 150 .

Inflation lexicale, 2 , p. 129,131

Information préalable à l'orientation, 1 , p. 49.

Inscriptions funéraires, 4, p. 280.

Inscrire (s') , 4, p. 309.

"Installation de traitement du courrier ", 2 , p. 150 .
Instrument de base du traducteur, 2, p. 129.

Instruments de musique, 1, p. 80 .

Intérêts, 4, p. 318.

Interprétation au Canada, 1, p. 39.

Interprète, 1, p. 36, 78 .

Interurbain automatique, 1, p. 32

Intraduisibilité, 1, p. 22.

Intraduisible, 2, p. 133.

Investissement, 4 , p. 317 .

Investissement en stocks, 4 , p. 317.

Investissement productif, 4, p. 317.

Investisseurs, 4, p. 317.

Jambe d'appel, 4, p. 312.

Jambe d'appui, 4, p. 311 .

Jambe d'attaque, 4, p. 311.

Jambe d'impulsion, 4, p. 312 .

Jambe libre, 4, p. 312.

Jaser, 1, p. 28.

Jargon administratif, 4, p. 324

Jargon atomique, 4 , p. 324

Jargon McLuhaniste, 4, p. 324.

Jargons, 2, p. 132.

Javelot, 4, p. 312 .

Jeux olympiques, 4 , p. 308

Joual, 1 , p. $28 ; 4$, p. 323 .

Lacunes, 1, p. 80 .

Lancer, 4 , p. 312 .

Lancer du disque, 4, p. 312.

Lancer du marteau, 4, p. 312.

Lancement du poids, 4, p. 312 .

Langue commune, 2, p. 129.

Langue de travail des traducteurs, 3, p. 180.

Langue écrite, langue parlée, 4, p. 331.

Langue et discours, 1, p. 14

Langue et pensée, 1, p. 11 .

Langue parlée, 4 , p. 330.

Latin, 1 , p. 80.

Lexicalisation des sigles, 4, p. 284.

Lexicologie comparée, 2, p. 130.

Lexique anglais-français (dérive des continents), 4 , p. 315 .

Liant dans une teinture, 3, p. 237.

Ligne droite, 4, p. 308.

Ligne du départ, 4, p. 309

Ligne opposée, 4 , p. 308.

Linguistique appliquée, 2 , p. 163 .

Linguistique du texte, 2, p. 165.

Linguistique et traduction, 1 , p. 108.

"Livraison spéciale », 1, p. 32

Livre, 4, p. 266

Livret d'opéra, 1, p. 80.

"Local ", 1, p. 32.

Local, 1, p. 30 .

Loi fédérale sur les langues officielles, 1, p. 40.

Loi 22,1, p. 59

Luge, 1, p. 31 .

Machines à trier, 2, p. 152

Madame le maire, 4 , p. 267

Magnétophone, 1, p. 66.

Magnétoscope, 2, p. 158.

Maillot, 4, p. 312 .

Maître d'armes, 2 , p. 142.

Mammifères, 1, p. 80.

Manuel de traduction, 4, p. 323.

Marche, 4, p. 312.

Marques d'indexation, 2, p. 151.

Matelas pneumatique, 4, p. 311 .

Médailles (inscriptions), 4, p. 280. 
Media, 1, p. 34

Mémoires en traduction, 1 , p. 80 .

Mener le train, 4, p. 310 .

Mesure du rendement, 3, p. 219.

Meta (statistiques), 4, p. 330

Métaphore, 3, p. 199.

Méthodologie, 1 , p. 48

Mise à la retraite anticipée, 3, p. 220.

Mise de fonds, 4, p. 318

Missions terminologiques, 1, p. 72

Mobilier, 1, p. 81.

Mobilité des cadres, 3 , p. 220

Modulation, 1, p. $80 ; 4$, p. 261.

Mordre, 4, p. 311 .

Morphèmes indifférenciés, 4, p. 264.

Motivation, 1, p. 35 .

Mots charnières, 4, p. 290.

Mots d'ambiance, 3 , p. 204

Mot-sentiment, 3, p. 204.

Mot-signe, 3, p. 204.

Mots-images, 3, p. 203

Mots-outils dans les sigles, 4, p. 288.

Moulinets, 4, p. 312.

Mousse, 4, p. 266

Multiplication des interprètes, 1, p. 39.

Musique, 4, p. 296.

Nacre, 4, p. 264

Nation, 1, p. 33.

National, 1, p. 32

Néologismes, 2, p. $131 ; 3$, p. $231 ; 4$, p. 269.

Neutre (en français), 4, p. 264

Niveaux expressifs, 3, p. 203

Noirceur, 1, p. 28

Nominaux, 4, p. 265.

Noms d'appareils, 4, p. 265.

Noms épicènes, 4 , p. 266.

Normalisation, 1, p. $68 ; 2$, p. $136 ; 4$, p. 333.

Normes de l'acier, 2, p. 167.

Normes d'examen, 1, p. 78 .

Normes en traduction, 1, p. 63

Notation des heures, 1, p. 32.

Note, 4, p. 319.

Notes tironiennes, 4, p. 281

Nuance, 2, p. 167.

Objectifs de l'enseignement, 1 , p. 45 .

Obligation de conversion, 3, p. 228.

Occasion (d'), 1, p. 31

"Octroi ", 1, p. 30

"Office ", 1, p. 30

Office de la langue française, 1, p. 34

Onde TE, 4, p. 296.

Onde TEM, 4, p. 296.

Onde TM, 4, p. 296.

Option d'achat, 3, p. 227

Optique expressive, $3, \mathrm{p} .194$

Optique métaphorique, 3, p. 199

Ordinateur, 4, p. 265

Orientation de l'enseignement, 1, p. 53.

Orthophonie et interprétation, 2, p. 166

Outillage de chasse aux mammiferes marins, 3, p. 237.

"Paletot ", 1, p. 29.

Parcours, 4, p. 311

Participations, 4, p. 317.

Particularités de formation des sigles, 4, p. 287.

"Partir ", 1, p. 29.

"Pas capable », 1, p. 29.

Passage, 4 , p. 310
Passe, 4, p. 311.

Pavillon, 1, p. 31

Pédagogie de la traduction, 1, p. 45,64 .

Peinture, 1, p. 81.

Peloton, 4, p. 310

Pelouse, 4, p. 308

Pensée et langue, 1, p. 11

Perche en fibre de verre, 4 , p. 312.

Perchiste, 4, p. 312.

Perfectionnement, 1, p. 52.

Perfectionnement des interprètes, 1, p. 41.

Performance, 1, p. 110

Personne (accord), 4, p. 265.

Pétrole, 1, p. 81.

Pied d'appel, 4, p. 311.

Piétiner devant l'obstacle, 4, p. 311.

Pige, 4, p. 327.

Pigistes, 1, p. 38

Piste, 4, p. 308

Piste d'élan, 4, p. 311

Phonétique et interprétation, 2, p. 166.

Phonostylistique, 1, p. 14.

Placement, 4, p. 317.

Planche d'appel, 4, 311.

Plan de carrière, 1 , p. $51 ; 3$, p. 220 .

Plongée sous-marine, 1 , p. 81 .

Plus-value, 3 , p. 227.

Poétesse, 4, p. 267.

Point abréviatif, 4, p. 283.

Point de piqué, 4 , p. 312.

Point de séparation, 4, p. 283.

Pointes, 4, p. 312.

Politique du bilinguisme, 1, p. 51.

Politique et traduction, 1, p. 110 .

Porte, 1 , p. 30

Portefeuilles-titres, 4, p. 318.

Porter un numéro de code sur, 2, p. 150.

Portrait-robot de l'étudiant, 1, p. 49.

Position du sujet de l'énonciation dans le discours, 1 , p. 111.

Possibilité d'enseigner la traduction, 1, p. 44.

Poste, 1, p. 32

Poteaux, 4, p. 311

Potentiel, 3, p. 219.

Potentiel de gestion, 3, p. 219

Pourcentage de participation, 4, p. 317.

"Poursuite ", 1, p. 29.

Pragmatique du texte, 2, p. 165.

Pratique de la rédaction, 1, p. 60 .

Préalable, 1, p. 31.

"Prégradué *, 1, p. 31

Préhistoire de la théorie de la traduction, 1, p. 8 .

Premier essai, 4, p. 311.

Prendre son élan, 4, p. 311.

Prépositions, 3, p. 241.

"Prérequis", 1, p. 31.

Prestation, 2, p. 155.

Prêts, 4, p. 309.

Primauté du concret, 3, p. 194.

Prime, 2, p. 155.

Principes de la traduction littéraire, 3, p. 238 .

Principes de rédaction, 1 , p. 62 .

Prises du témoin, 4, p. 311 .

Prix de rachat, 3 , p. 228.

Prix de traduction, 3, p. 245. 
Problèmes de choix des investissements, 4, p. 317.

Procédés d'abréviation des mots, 4, p. 271.

Procédés de traduction, 1, p. 9.

Processus traductionnel, 1, p. 107.

Produit fini, 1, p. 20, 50

Produits sidérurgiques, 2, p. 167

Profession et université, 1, p. 57, 63

Professionnel et amateur, 1, p. 45.

Professionnels et usagers, 4, p. 329.

Programmes d'enseignement, 1, p. 43.

Programmes de placement externe, 3, p. 220.

Prolifération du lexique, 2, p. 132.

Prononciation des sigles, 4 , p. 283.

Province, 1, p. 32

Provincial, 1, p. 32

Publicité, 1, p. 65, 80, 82.

"Pupitre de codage ", 2, p. 151.

Pupitre d'indexation, 2, p. 151.

"Qualification ", 3, p. 219.

Qualité, 1, p. 50

Qualités professionnelles du traducteur, 1, p. 51 .

Quantité, 1, p. 50.

Quart (notation des heures), 1, p. 32.

Quarts de finale, 4, p. 309

Quatre cents mètres haies, 4 , p. 310 .

Quatre $X$ cents mètres, 4, p. 311 .

Québec (Qué. ou P.Q.), 1, p. 32

Québec et francophonie canadienne, 4 , p. 328.

Québec et traduction, 4, p. 331

Québéciser, 1, p. 33 .

Québécitude, 1, p. 33

Québécois, 1, p. 33.

Quelqu'un (accord en genre), 4, p. 265

Quittance, 4, p. 319.

Racines culturelles du Québécois. 4, p. 331.

Rapport qualité/quantité, 1, p. 50.

Réaffectation, 3, p. 220.

Réception, 1, p. 30.

"Réclamation », 1, p. 30.

Reconnaissance professionnelle, 1, p. 62 , 65,68 .

Recyclage, 1, p. 52.

Recyclage des interprètes, 1 , p. 41.

Rédaction, 1, p. 65.

Refrancisation, 1, p. 29.

Régie, 1, p. 33

Relations de/du travail, 3, p. 207.

Relations industrielles, $3, \mathrm{p}, 207$.

Relations ouvrières, 3 , p. 207.

Relations professionnelles, 3, p. 207.

Relations syndicales, 3 , p. 208.

Relayé, 4, p. 311.

Relayeur, 4, p. 311.

Relève, 3, p. 220.

Relevé de compte, 4 , p. 319

Remboursement anticipé, 3, p. 227.

Rencontre, 4, p. 308.

Renouvellement de la créativité, 3 , p. 219

Renouvellement du talent créateur, 3, p. 218.

Repère, 4, p. 311.

Réseau mondial de banques de terminologie, 4 , p. 330

Résistance, 4, p. 310.

Responsable de la francisation, 4, p. 329.

Ressources humaines, 3, p. 217.
Revalorisation de la langue maternelle, 1, p. 62.

Réviseur, 1, p. 51 ; 2, p. 156.

Révision (tarif), 4, p. 327.

Révision comptable, 2, p. 156, 157.

Revues de traduction, 3, p. 181.

Retraduction, 2, p. 136.

Rétribution, 3, p. 218.

Rôle de conseil du traducteur, 1, p. 51.

Rouleau californien, 4, p. 311 .

Rouleau ventral, 4, p. 311 .

"Royautés ", 1, p. 30.

Rivière, 4, p. 311 .

Saturation par la traduction, 1, p. 64.

Saut à la perche, 4, p. 312.

Saut en hauteur, 4 , p. 311 .

Saut en longueur, 4, p. 311

Sautoir, 4, p. 311 .

Science de la traduction, 2, p. 164

Sciences de la communication, 1, p. 12

Second souffle, 4, p. 310 .

Secours, 2, p. 155 .

Sélection des candidats, 1, p. 61 .

Sémantique, 2, p. 165.

Séries, 4, p. 309.

Service de recherche, 2, p. 133.

Service de traduction dans l'entreprise, 1, p. 111 .

Serrée, 4 , p. 309.

Sexiste, 4, p. 263.

Sigle, 4, p. 271, 275, 277.

Sigle (étymologie), 4, p. 278

Sigles expressifs, 3, p. 195

Sigles généraux, 4, p. 301

Sincérité, 2, p. 156.

Sinistre, 1, p. 30 .

Société, 1, p. 33.

Société des diplômés de l'Institut de traduction, 1, p. 76.

Société des traducteurs de Montréal, 1, p. 75.

Société des traducteurs du Québec, 3, p. 247.

Société des traducteurs et interprètes du Canada, 1, p. 76.

Sociétés professionnelles, 1, p. 63.

Solutions obliques de traduction, 2, p. 133.

Sondage auprès des lecteurs, 3 , p. 179.

Sondages, 2 , p. 156

Sons et bruits, 1, p. 80

Sortie du virage, 4 , p. 309

Soufflet, 3 , p. 237

Souffleur de verre, 4 , p. 270.

Spécialisation, 2, p. 130.

Sprint contrôlé, 4, p. 310 .

Stade, 4, p. 308 .

Stage, 1, p. 55

Stagflation, 2, p. 132

Stagiaire, 1, p. 55 .

Statistiques de traduction, 2 , p. 163.

Sténogramme, 4, p. 279.

Style, 1, p. 13 .

Style dorsal, 4, p. 312 .

Stylistique, 1, p. 14

Stylistique comparée, 1 , p. 12,14 ; 3, p. 194

Subjectivisme langagier, 2, p. 136.

Subside, 2, p. 155 .

Substantifs vidés de leur sens, 2, p. 134.

Subvention, 1 , p. $30 ; 2$, p. 155 .

Suppression des points abréviatifs, 4, p. 285. 
Suspensoir, 4, p. 312.

Survêtement, 4, p. 308.

Symbole, 4, p. 275,295

Synonymie, 3, p. 206.

Systèmes abréviatifs, 4 , p. 282.

Tabac, 1, p. 81

Tableau d'affichage, 4, p. 308.

Tableau récapitulatif de l'étude sur le genre, 4, p. 268

Talent créateur, 3 , p. 218

Tarif à la pige, 4, p. 327.

Tarif horaire, 4, p. 327.

Tarif minimal de traduction 4, p. 327.

Tarif-révision, 4, p. 327.

Tartan (en) , 4, p. 308.

Taux de consommation de l'oxygène, 4 , p. 310.

Taux de désistement, 1, p. 52.

Taux de rotation du personnel, 1, p. 52 .

Technicité des textes, 1, p. 60.

Technique de rédaction, 1, p. 49.

Techtonique des plaques, 4 , p. 313.

Techtonique globale, 4, p. 313 .

Témoin, 4, p. 311 .

Temps, 4, p. 309.

Temps de passage, 4 , p. 310 .

Termes comptables, 1 , p. 81

Termes journalistiques, 1 , p. 81

Termes préfabriqués, 4, p. 291.

Terminologie, 1, p. 58, 71 .

Terminologie (colloque de), 4, p. 332.

Terminologie dans l'entreprise, 1, p. 71

Terminologie (définition), 4, p. 333.

Terminologie de traduction, 1, p. 72.

Terminologie et sémantique, 2, p. 165 .

Terminologies, 2, p. 132

Terminologies particulières, 2, p. 130.

Terminologue d'entreprise, 1 , p. 73.

Textologie comparée, 2, p. 164.

Théorie de la traduction, 1, p. $7,13,54$; 2 , p. 132 .

Théorie de l'interprétation, 2, p. 164.

Théories linguistiques de la traduction, 1, p. 10 .

Tilde, 4, p. 281

Titres, 4 , p. 318

Titres et grades, 1, p. 81 .

Toponymie, 1 , p. 80 .

Tour de cloche, 4 , p. 310 .

Tour de piste, 4, p. 310.

Tours, 4 , p. 312

Traducteur à la pige, 3 , p. 246

Traducteur-artisan, 2, p. 129.

Traducteur, le, 1, p. 75 .

Traducteurs anglophones, 1, p. 60.

Traducteurs et interprètes, 2, p. 163.
Traducteurs littéraires, 1, p. $69 ; 2$, p. 175 ; 3, p. 244.

Traduction au magnétophone, 1, p. 66, 67

Traduction automatique, 1, p. 19, 54, 106.

Traduction de la poésie, 3, p. 229.

Traduction des lois, 1, p. 69.

Traduction des sigles, 4, p. 292.

Traduction de textes \& informatifs » ou documentaire, 1 , p. 65 .

Traduction et entreprise, 3, p. 240.

Traduction et stylistique comparée, 1, p. 18.

Traduction et théorie, 1, p. 21.

Traduction, exercice littéraire, 1, p. 15.

Traduction littéraire, 1, p. 11.

Traduction médicale, 1, p. 80 .

Traduction symbolique, 1, p. 63 .

Traduction utilitaire, 1, p. 63 .

Traduction vers l'anglais, 1, p. 59.

Traductologie, 2, p. 163.

Traduisible et intraduisible, 2, p. 133.

Train, 4, p. 310.

Traitement des traducteurs, 3, p. 180.

Trait sémantique, 4, p. 264.

Translation d'un message, 1, p. 10.

Transmettre, 4, p. 311 .

Transmissions, 4, p. 311.

Transposition, 1, p. 80 .

Travaux publics, 2 , p. 170

Tribunes, 4, p. 308.

Tri du courrier, 2, p. 150.

Triple saut, 4, p. 312.

Unilinguisme, 4, p. 322.

Union des traducteurs québécois, 1, p. 77.

Université et profession, 1, p. 57.

Urgence (tarif), 4, p. 327.

Usage, 4 , p. 325.

Valeur distinctive de certains féminins, 4, p. 267.

Valeurs mobilières, 3 , p. 225.

Variation des stocks, 4, p. 317.

Vérificateur, 2, p. 156.

Vérificateur interne, 2, p. 157.

Vérifications matérielles, 2, p. 156

Ville, 1, p. 33

Virages, 4 , p. 308

Vocabulaire francais-anglais de l'escrime, 2 , p. 148.

Voile, 4 , p. 266

Vue, 3, p. 194

Vue concrète, 3, p. 203.

Vue directe, 3, p. 199.

Vue émotive, 3, p. 195, 203.

Vue oblique, 3, p. 199.

Vue représentative, 3, p. 195, 203.

Zoïles, 3, p. 235.

Zone de transmission, 4, p. 311. 